\title{
BIBLIOGRAPHY
}

1. G. Kreisel, Relative consistency and translatability (abstract), J. Symbolic Logic 23 (1958), 108-109.

2. J. Myhill, Creative sets, Math. Logik Grundlagen Math. 1 (1955), 97-108.

3. R. Smullyan, Theory of formal systems, Annals of Mathematics Studies No. 47, Princeton Univ. Press, Princeton, N. J., 1961.

4. A. Tarski, A. Mostowski and R. Robinson, Undecidable theories, North-Holland, Amsterdam, 1953.

InSTITUTE for Advanced Study and

UNIVERSITY OF MINNESOTA

\section{REPORT ON ATTAINABILITY OF SYSTEMS OF IDENTITIES}

\author{
BY T. TAMURA
}

Communicated by Edwin Hewitt, December 7, 1964

1. Introduction. This note is to report the main results in the paper, Attainability of systems of identities on semigroups, which will be published elsewhere with detailed proof.

Let $f$ and $g$ be words, i.e., finite sequences of letters. By an identity we mean an equality $f=g$ of two words $f$ and $g$. Let $J$ be a system of identities $T_{\lambda}$,

$$
\mathfrak{J}=\left\{T_{\lambda} ; \lambda \in \Lambda\right\} \quad \text { where } T_{\lambda} \text { is " } f_{\lambda}=g_{\lambda},
$$

for example, $\left\{x y z=x z y, x=x^{2}\right\},\left\{x y=y x, x=x^{2}\right\}$ and so on.

Let $S$ be a semigroup. For a fixed $S$ and a fixed J, consider the set $\mathcal{e}$ of all congruences $\rho$ on $S$ such that $S / \rho$ satisfies $\mathfrak{I}$, in other words, $\mathfrak{J}$ identically holds if all letters are replaced by elements of $S / \rho$. There is the smallest element $\rho_{0}$ in $\mathcal{C}$ in the sense that $\rho_{0} \subseteq \rho$ for all $\rho \in \mathcal{C}$ $[1],[4],[7],[8],[9],[11]$. Then $\rho_{0}$ is called the smallest J-congruence, and the partition of $S$ due to $\rho_{0}$ is called the greatest J-decomposition. Of course, such a decomposition of $S$ is unique. If the cardinal number $\left|S / \rho_{0}\right|$ of $S / \rho_{0}$ is greater than 1 , then $S$ is called J-decomposable; if $\left|S / \rho_{0}\right|=1$, then $S$ is J-indecomposable. In particular, if $J$ is a semilattice, that is, $J=\left\{x=x^{2}, x y=y x\right\}$, then $\rho_{0}$ is called the smallest semilattice-congruence or, simply, s-congruence. The author proved in his papers [8], [10] the following theorem, and also Petrich recently proved the equivalent statement [6]. 
THEOREM 1. In the greatest s-decomposition of a semigroup, each congruence class, which is therefore a subsemigroup, is s-indecomposable.

Thus any semigroup is a semilattice of s-indecomposable semigroups, namely, the set union of disjoint subsemigroups of special type, "s-indecomposable," and the factor semigroup is a semilattice. Completely (o-) simple semigroups (with zero-divisor), their direct products, and nilpotent semigroups are examples of s-indecomposable semigroups. This theorem actually contributes much to the study of structure and construction theory of semigroups. However, the theorem is based on s-decomposition. There might exist a Jdecomposition which is different from an s-decomposition such that a congruence class - if it is a subsemigroup-is 3 -indecomposable. If this assumption were true, we could study the construction theory of semigroups from a different point of view.

Does there exist such a $J$ besides a semilattice? Professor A. H. Clifford proposed this question in his letter to the author in 1963. In this note the author gives a negative answer to this question as far as the systems $\mathfrak{J}$ of identities are concerned.

2. Definitions and theorems. Let $J$ be a system of identities, $\subseteq$ be a family of semigroups, and let $\xi_{S}$ be the smallest J-congruence on $S \in \mathfrak{S}$. $\Im$ is called attainable on all semigroups of $\mathfrak{S}$ (or simply "on $\mathfrak{S}$ ") if, for each $S \in \subseteq$, the following condition is satisfied:

If a congruence class of $S$ modulo $\xi_{S}$ is a subsemigroup, then it is 7 -indecomposable.

In particular, if $\subseteq$ is the family of all semigroups (without restriction) and if $J$ is attainable on $\mathfrak{S}, J$ is called attainable (on all semigroups). If $J$ is attainable on $\mathfrak{S}$ and if $\mathfrak{S}$ consists of a semigroup $S$ alone, we say that $\mathcal{J}$ is attainable on a semigroup $S$.

Two systems $\mathfrak{J}$ and $\mathcal{S}$ of identities are called equivalent on all semigroups of $\subseteq$ if $\mathfrak{J}$ implies $\mathcal{S}$ and $\mathcal{S}$ implies $\mathfrak{J}$ under the condition that all the letters are elements of any semigroup of $\subseteq$, where, if $\subseteq$ is the family of all semigroups, we say that $\zeta$ and $\delta$ are equivalent.

As the special cases, if $J$ is equality, that is, equivalent to $\{x=x\}$, or if $J$ is universality, that is, equivalent to $\{x=y\}$, then $J$ is attainable on all semigroups. $J$ is called trivial if $J$ is either equality or universality. If $J$ is equivalent to equality or universality on all semigroups of $\mathfrak{S}, \mathfrak{J}$ is called equality or universality on $\mathfrak{S}$.

In the previous paper [8], it was shown that neither $\left\{x=x^{2}\right\}$ nor $\{x y=y x\}$ is attainable on all semigroups. In the present note we have the following main theorems: Let $J$ be nontrivial through the theorems below. 
THEOREM 2. $J$ is attainable on all semigroups if and only if $\mathfrak{J}$ is equivalent to $\left\{x=x^{2}, x y=y x\right\}$.

THEOREM 3. I is attainable on all commutative semigroups if and only if $J$ is equivalent to $\left\{x=x^{2}\right\}$.

THEOREM 4. $J$ is attainable on all bands if and only if $J$ is equivalent to $\{x y=y x\}$ on all bands.

TheOREM 5. No nontrivial system of identities is attainable on all groups, nor on all abelian groups.

3. Remark and problems. A groupoid is a system with a binary operation. Let $J$ be a system of identities $T_{\lambda}$ where the concept of words $f_{\lambda}$ and $g_{\lambda}$ must be modified a little, for example, a word $(x y)(x y)$ is different from $x\{(y x) x\}$, and so on. It is still true that there is a smallest 3 -congruence on any groupoid [1], [4], [9]; in particular, any groupoid has a smallest s-congruence.

We can show the following results by simple counterexamples.

(5) $\{x y=y x\}$ is not attainable on all idempotent groupoids.

(6) $\left\{x=x^{2}\right\}$ is not attainable on all commutative groupoids.

(7) $\left\{x=x^{2}, x y=y x\right\}$ is not attainable on all groupoids.

(8) $\{(x y) z=x(y z)\}$ is not attainable on all groupoids.

The following problem is still open.

Problem 1. Is there any nontrivial attainable system of identities on all groupoids?

So far all the letters in each identity are regarded as variables.

PRoBlem 2. If an identity admits constant elements, what about the problem of attainability of a system of identities on all semigroups? In this case the sense of attainability has to be modified.

We can consider identities $f_{\lambda}=g_{\lambda}$ on algebraic systems with more than one binary operation. In such a case, the words $f_{\lambda}, g_{\lambda}$ are interpreted as sequences of letters such that they are connected by the operations and parentheses.

Problem 3. Find attainable systems of identities on all rings or lattices.

From another point of view, the following problems are raised:

Problem 4. Let $\delta$ be a system of implications:

$$
f_{\lambda \mu}=g_{\lambda \mu}, \quad \mu \in \mathrm{M}, \Rightarrow h_{\lambda}=k_{\lambda}, \quad \lambda \in \Lambda,
$$

where $f_{\lambda \mu}, g_{\lambda \mu}, h_{\lambda}$ and $k_{\lambda}$ are words which may contain a constant element. Under what condition on $S$ is $S$ attainable on all semigroups?

Of course, in the case where constant elements are contained, we need the same consideration as in Problem 2. The condition "weak reductivity" [1] is attainable. 
In this note we have studied the condition on a fixed system $J$ of identities under which $J$ is attainable on all semigroups. On the other hand:

Problem 5. Let a system $\mathfrak{J}$ and a semigroup $S$ be fixed. Under what condition on $J$ and $S$ is $J$ attainable on the semigroup $S$ ?

\section{REFERENCES}

1. A. H. Clifford and G. B. Preston, Algebraic theory of semigroups, Vol. 1, Math. Surveys No. 7, Amer. Math. Soc., Providence, R. I., 1961.

2. N. Kimura, Note on idempotent semigroups. III, Proc. Japan Acad. 34 (1958), 113-114. 123.

3. - Note on idempotent semigroups. IV, Proc. Japan Acad. 34 (1958), 121-

4. - On some existence theorems on multiplicative systems. I, Proc. Japan Acad. 34 (1958), 305-309.

5. - The structure of idempotent semigroups (1), Pacific J. Math. 8 (1958), 257-275.

6. M. Petrich, The maximal semilattice decomposition of a semigroup, Bull. Amer. Math. Soc. 69 (1963), 342-344.

7. T. Tamura and N. Kimura, Existence of greatest decomposition of a semigroup, Kōdai Math. Sem. Rep. 7 (1955), 83-84.

8. T. Tamura, The theory of construction of finite semigroups. I, Osaka Math. J. 8 (1956), 243-261.

9. - Operations on binary relations and their applications, Bull. Amer. Math. Soc. 70 (1964), 113-120.

10. - Another proof of a theorem concerning the greatest semilattice-decomposition of a semigroup, Proc. Japan Acad. 40 (1964), 777-780.

11. M. Yamada, On the qreatest semilattice decomposition of a semigroup, Kōdai Math. Sem. Rep. 7 (1955), 59-62.

12. M. Yamada and N. Kimura, Note on idempotent semigroups. II, Proc. Japan Acad. 34 (1958), 110-112.

13. M. Yamada, The structure of separative bands, Dissertation, University of Utah, Salt Lake City, Utah, 1962.

University of California, Davis 